\title{
EchoGéo
}

$9 \mid 2009$

L'Asie centrale : le temps des recompositions

\section{La ville du Sud en temps réel}

De l'utilité de la photographie aérienne sous cerf-volant dans les études urbaines

Benjamin Bosselut, Marion Broquère, Armelle Choplin et Simon Nancy

\section{OpenEdition}

\section{Journals}

Édition électronique

URL : https://journals.openedition.org/echogeo/11305

DOI : $10.4000 /$ echogeo. 11305

ISSN : 1963-1197

Éditeur

Pôle de recherche pour l'organisation et la diffusion de l'information géographique (CNRS UMR 8586)

Référence électronique

Benjamin Bosselut, Marion Broquère, Armelle Choplin et Simon Nancy, « La ville du Sud en temps réel », EchoGéo [En ligne], 9 | 2009, mis en ligne le 10 juillet 2009, consulté le 01 août 2021. URL: http://journals.openedition.org/echogeo/11305; DOI : https://doi.org/10.4000/echogeo.11305

Ce document a été généré automatiquement le 1 août 2021

EchoGéo est mis à disposition selon les termes de la licence Creative Commons Attribution - Pas d'Utilisation Commerciale - Pas de Modification 4.0 International (CC BY-NC-ND) 


\section{La ville du Sud en temps réel}

De l'utilité de la photographie aérienne sous cerf-volant dans les études urbaines

Benjamin Bosselut, Marion Broquère, Armelle Choplin et Simon Nancy

Photo 1 - Front d'urbanisation au nord de Nouakchott

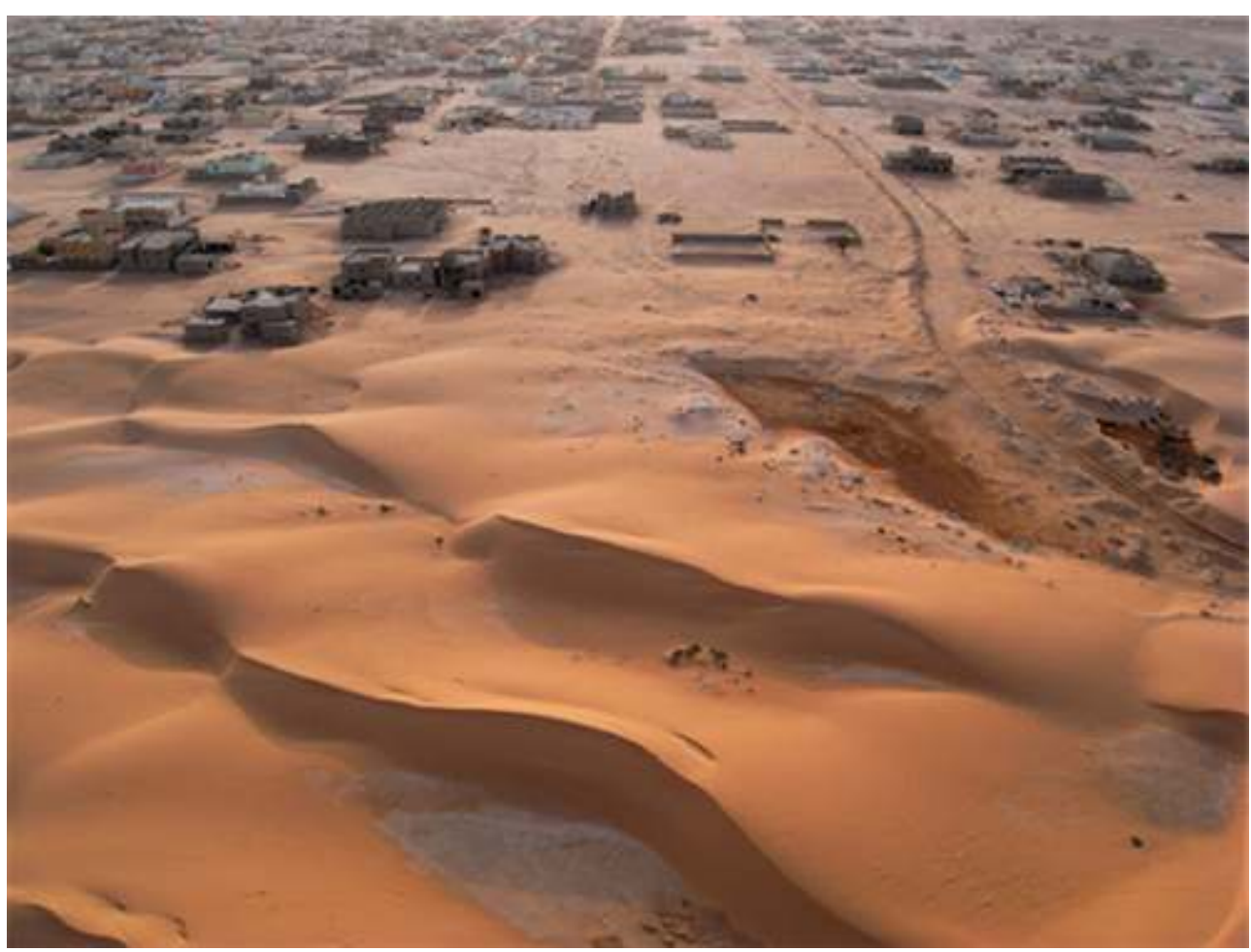

Cliché « En Haut ! », Tous droits réservés, Nouakchott, Mauritanie, Novembre 2008 
Photo 2 - Front d'urbanisation au nord-ouest de Nouakchott

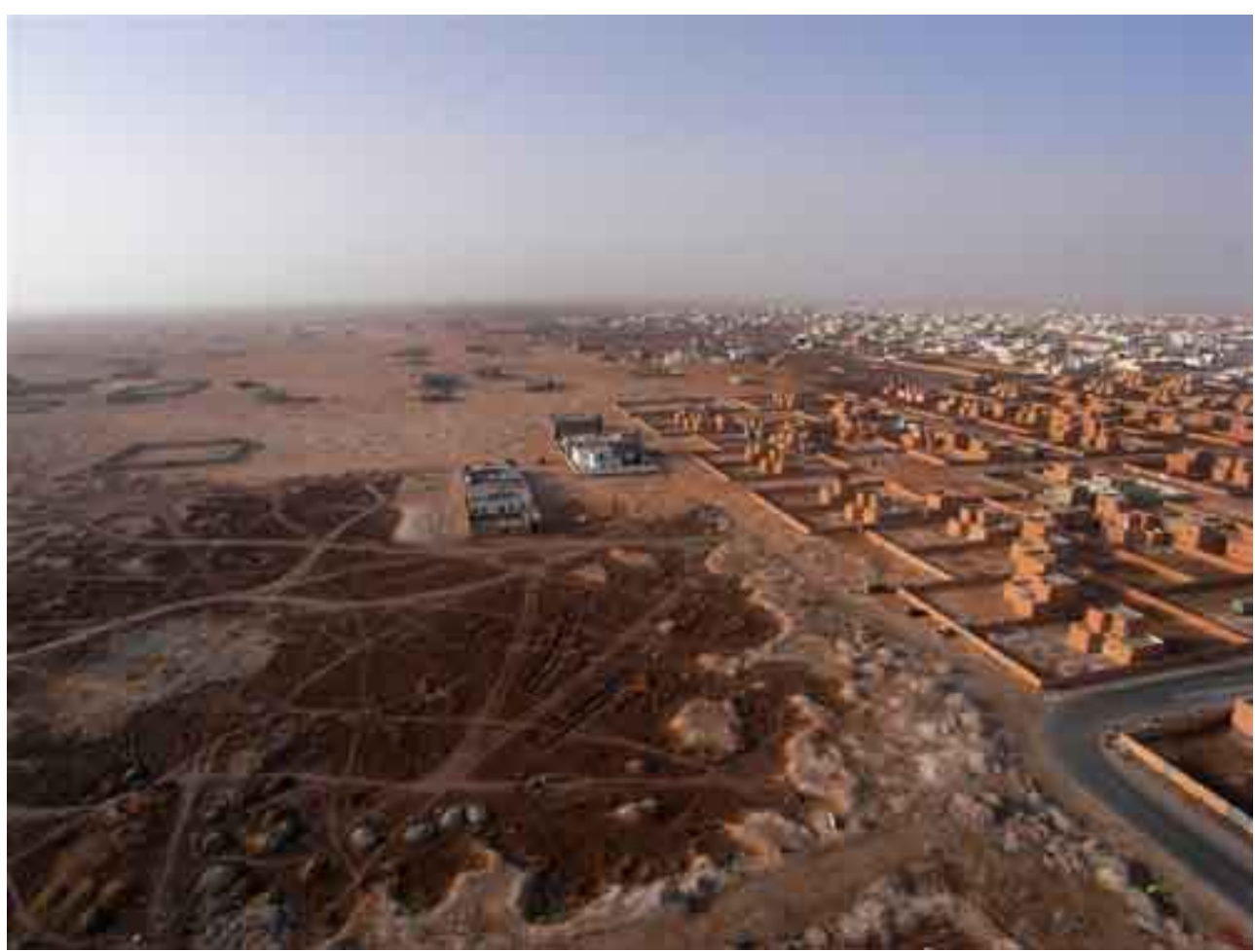

Cliché « En Haut! », Tous droits réservés, Nouakchott, Mauritanie, Novembre 2008

Photo 3 - Front d'urbanisation au sud-ouest de Nouakchott

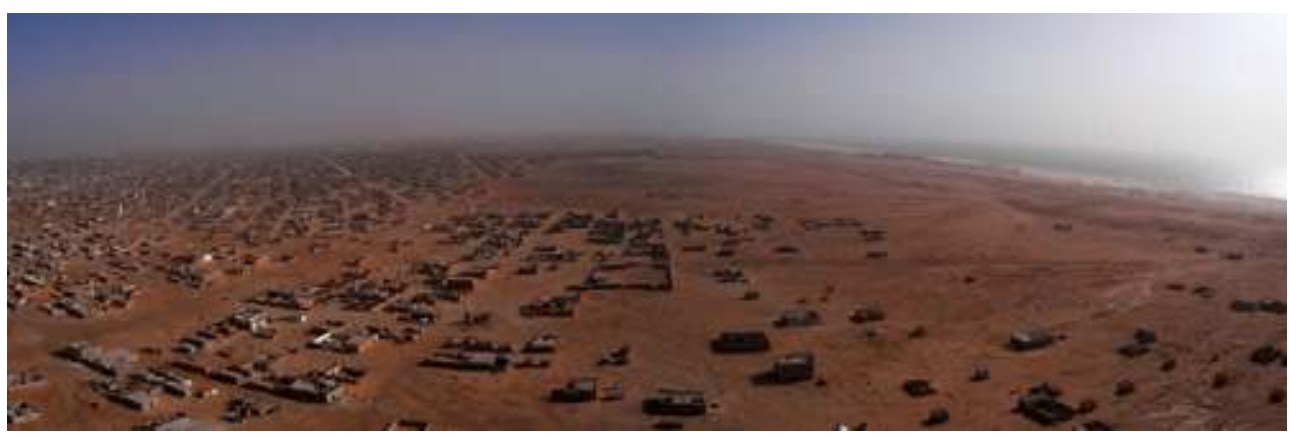

Cliché « En Haut! », Tous droits réservés, Nouakchott, Mauritanie, Novembre 2008

\section{Comment évaluer un programme d'habitat social dans une ville en mouvement?}

1 Tel Icare, le chercheur qui travaille sur la ville se prend souvent à rêver de voler, ou du moins survoler son objet d'étude afin de voir d'en haut ce qu'il ne parvient à saisir d'en bas. Depuis peu, Google Earth lui permet de caresser en partie ce rêve, de façon fictive et pour quelques villes seulement. S'il lui vient le souhait de s'intéresser aux villes dites du «Sud », qui connaissent une croissance rapide, cela devient plus problématique. La prise est bien souvent floue et rarement mise à jour. Comment alors voir cette ville du "Sud» dont la réalité change d'heure en heure ? Comment visualiser ces mutations parfois brutales qu'elle subit? Comment encore rendre compte de l'avancée quasi 
quotidienne des fronts urbains alors mêmes que les cartes réalisées localement sont souvent de mauvaise qualité et guère actualisées, faute de moyens? La photographie aérienne sous cerf-volant offre un outil susceptible d'apporter quelques réponses à ces questions puisqu'elle permet une couverture en «temps réel». Sa flexibilité dans l'usage, le moindre coût qu'elle propose et son aspect écologique en font un outil particulièrement intéressant. Plus encore, elle livre immédiatement nombre d'informations. Par sa forte capacité heuristique, en particulier à l'échelle micro, le document produit peut s'exploiter de façon brute ou bien servir de base à une analyse scientifique plus poussée. Nous avons pu découvrir et expérimenter quelques unes de ces possibilités dans le cadre d'une étude conduite dans la ville de Nouakchott, capitale de la Mauritanie. Nouakchott illustre parfaitement les phénomènes d'urbanisation rapides et incontrôlés puisque cette capitale, créée ex-nihilo en 1957 où elle n'était alors qu'un vulgaire puits autour duquel évoluaient quelque 800 nomades, compte aujourd'hui près de 800000 habitants (Choplin, 2009).

Figure 1 - Croissance spatiale de Nouakchott (1950-2008)

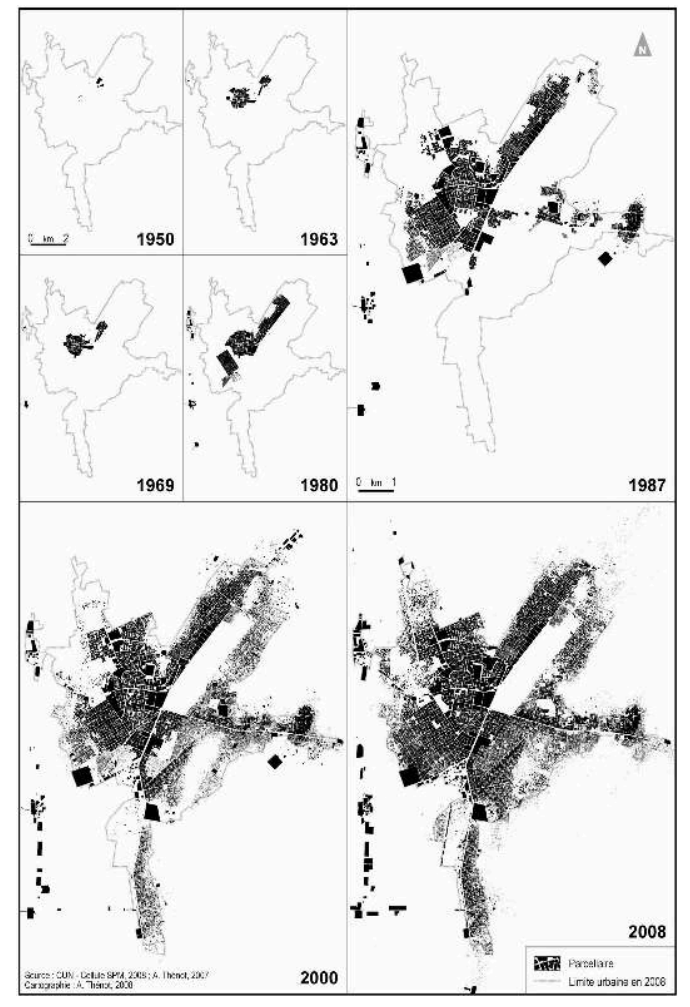

Source : d'après A. Thénot, 2007, in A. Choplin, 2009

2 Nous avons été chargés de réaliser l'étude d'impact d'un programme d'habitat social mis en œuvre par une ONG française, le GRET (Groupe d'Echange et de Recherche Technologique) ${ }^{1}$. Ce programme, dénommé Twize, avait pour objectif de permettre l'accès à l'habitat en dur aux populations pauvres des bidonvilles et des quartiers non équipés. Entre 2000 et juin 2008 (date de la fin du programme), quelque 6500 modules ont été construits (majoritairement des chambres de $20 \mathrm{~m}^{2}$ ), dans sept quartiers de la capitale et à Nouadhibou, deuxième ville du pays ( $(\mathcal{f}$. photo 5$)$. Un système couplé de microcrédit et de subvention étatique a permis l'obtention de ce module, dont le prix 
est estimé à environ 300000 ouguiyas (soit près de 1000 euros) (Creusot, 2002 ; GRET, 2003 ; Choplin, 2006).

Photo 4 - Habitat précaire dans le bidonville avant le programme Twize

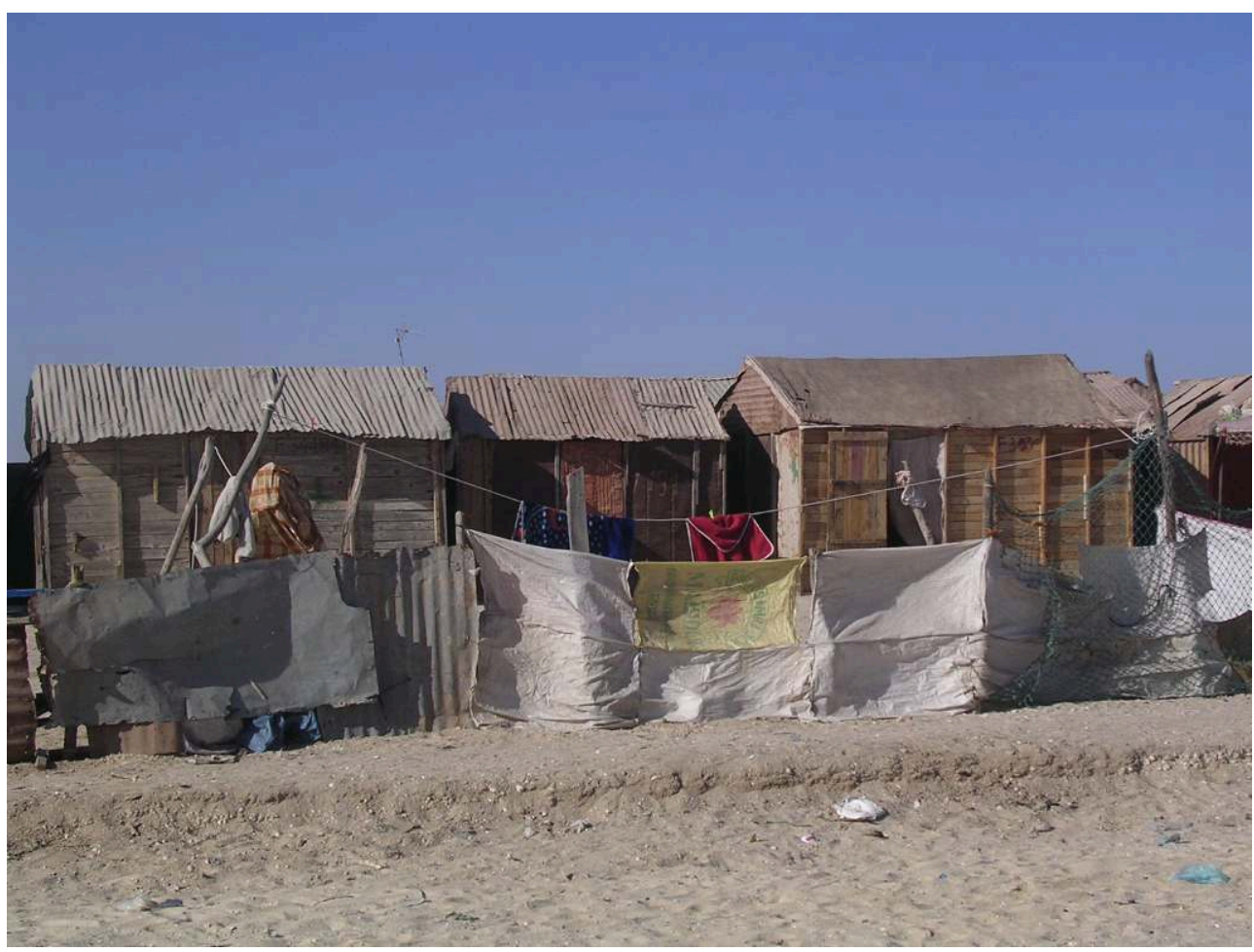

Cliché d'Armelle Choplin, Nouakchott, novembre 2003 


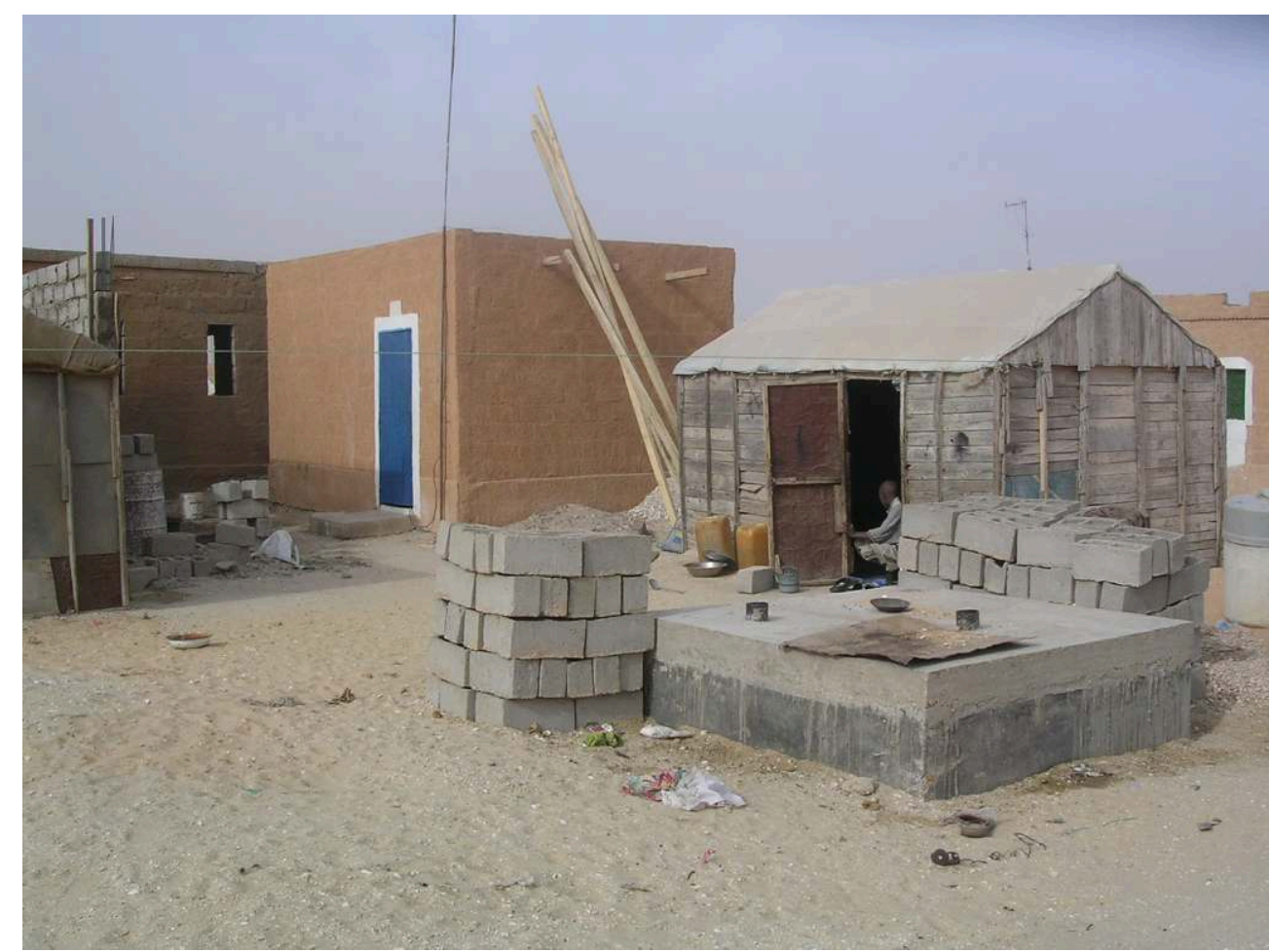

Cliché d'Armelle Choplin, Nouakchott, novembre 2003

3 L'étude d'impact que nous avons menée entre septembre 2008 et janvier 2009 devait rendre compte des transformations induites (in)directement par le programme. Un volet portait en particulier sur l'évolution des quartiers d'intervention, hier encore caractérisés par l'habitat précaire (tentes, baraques en bois). Les mandataires de l'étude souhaitaient que soit évaluée la «durcification» (processus de transformation d'un habitat majoritairement précaire à un habitat en dur) et, pour cela, que soit mise au point une méthode permettant d'évaluer de visu et de calculer l'impact du projet sur ce phénomène ${ }^{2}$. Comment rendre compte des transformations ? Etaient-elles à rapprocher du programme ou d'ordre spontané ? Le programme a-t-il insufflé ou bien accéléré ce processus de « durcification »?

4 Un problème de taille se posait alors : comment évaluer concrètement ces changements survenus pour certains en quelques mois, alors que plans et autres schémas font cruellement défaut en Mauritanie en général et à Nouakchott en particulier (couverture aérienne à jour manquante, absence de cadastre et de carte détaillée à l'échelle intra-urbaine) ? Nous n'avions à notre disposition que la couverture aérienne de Nouakchott en 2000 disponible au laboratoire PRODIG (UMR 8586) et les images satellites libres de droit qu'utilise Google Earth qui datent dans le cas mauritanien de 2005, difficilement exploitables à l'échelle des quartiers (manque de précision).

La rencontre avec le collectif «En Haut!» qui expérimente la photographie aérienne sous cerf-volant en Mauritanie permit de dépasser ces blocages initiaux. Dans le cadre de cette étude, ce type de photographie aérienne est devenue un outil rapide, fiable, économique, exploitable dans un laps de temps court et de différentes manières, et notamment scientifique. La technique utilisée a non seulement permis d'actualiser nos données mais d'identifier les mutations, voire de les mesurer. 


\section{Technique de prise de vue}

6 La photographie aérienne sous cerf-volant consiste à envoyer dans les airs un appareil photo porté par une nacelle mobile fixée au fil d'un cerf volant. L'utilisation de ce principe est ancien et attesté dès 1888, année où Arthur Batut fit voler une chambre noire sous un grand cerf-volant (Batut, 1890). Aujourd'hui cette technique, connue sous le nom de Kite Aerial Photograpy (KAP), tend à se diffuser et suscite même un regain d'intérêt grâce au matériel utilisé, de plus en plus fiable. Au delà de son utilisation ludique, le KAP à fait ses preuves dans diverses applications, en particulier l'archéologie (Anderson, 1979; Ales, Tagala, 1985) et le suivi des états de surface (Tielkes, 2003).

\section{Préparer le vol}

7 Au préalable de la prise de vue proprement dite, il s'avère essentiel de définir une méthodologie d'étude car la technique du cerf-volant ne permet pas de photographier un quartier dans son intégralité. Il convient alors de réaliser un premier diagnostic territorial afin de sélectionner quelques secteurs, de définir en quelque sorte des « extraits » de territoire à photographier.

Dans le contexte de l'étude d'impact du programme Twize, des échantillons de quartiers où l'ONG est intervenue ont été identifiés. Pour trouver un panel représentatif, nous avons été particulièrement attentifs aux éléments structurant l'urbanisation. Ces derniers diffèrent selon les zones en fonction de l'histoire du quartier (zone plus ou moins récente), de la présence et de la proximité des équipements et infrastructures (école, fontaine, mosquée, route goudronnée, électricité...). Nos choix ont donc veillé à diversifier ces critères ( $C f$. figure 2). Par ailleurs, pour faciliter les prises, nous avons eu tendance à choisir des secteurs situées à proximité, de façon à limiter les atterrissages et par conséquent le temps de vol. 


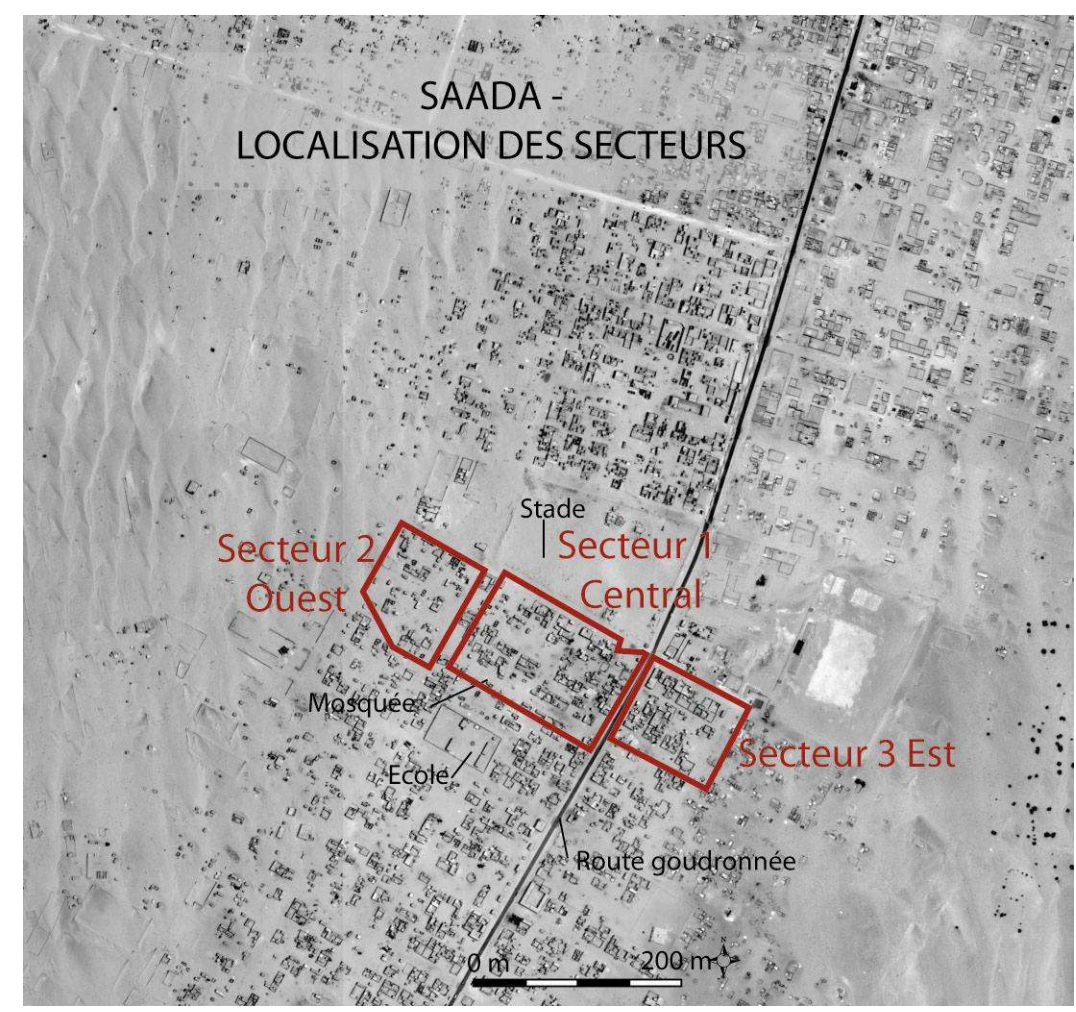

Dans le quartier de Saada, nous avons choisi de survoler les secteurs à proximité du stade, de l'école et de part et d'autres du goudron.

Cette reconnaissance des zones a d'abord été effectuée à partir de plans, de cartes et de données urbaines disponibles, puis complétée par un déplacement sur le terrain pour repérer les espaces à survoler. L'objectif premier est de voir les possibilités offertes pour organiser le vol. Si la Mauritanie offre des conditions optimales en termes de vent et de lumière, cette technique présente néanmoins un certain nombre de contraintes en milieu urbain. Les infrastructures, et en particulier les lignes électriques restreignent les déplacements. Les survols à proximité d'un aéroport, de zones militaires ou de prisons sont à éviter et les aléas climatiques (vents de sable, rafales, chutes de vent), ainsi que le risque omniprésent d'une chute du matériel dans une zone densément peuplée sont à prendre en compte. Au final, il faut identifier autant le secteur à photographier, que les aires de décollage et d'atterrissage, ainsi que le circuit dégagé d'obstacle que la personne dirigeant le cerf-volant doit parcourir.

\section{Déroulement d'une séance de prise de vue}

Après avoir étudié le sens et la force du vent, le cerf volant est envoyé dans les airs jusqu'à une altitude où le vent est stable. Sur le fil, on suspend alors une nacelle sur laquelle est fixé l'appareil photo numérique. L'ensemble reste horizontal grâce à un judicieux jeu de poulie appelé " Picavet ". Le grand angle, qui permet de couvrir plus de surface, est utilisé en priorité. Le déclenchement et les rotations verticales et horizontales de l'appareil s'opèrent depuis le sol grâce à un système de radiocommande. Ce matériel permet ainsi de réaliser des prises de vues obliques et verticales ( $C f$. photos 6 et 7$)$. 
Photo 6 - Prise de vue oblique du quartier de Dar El-Beïda

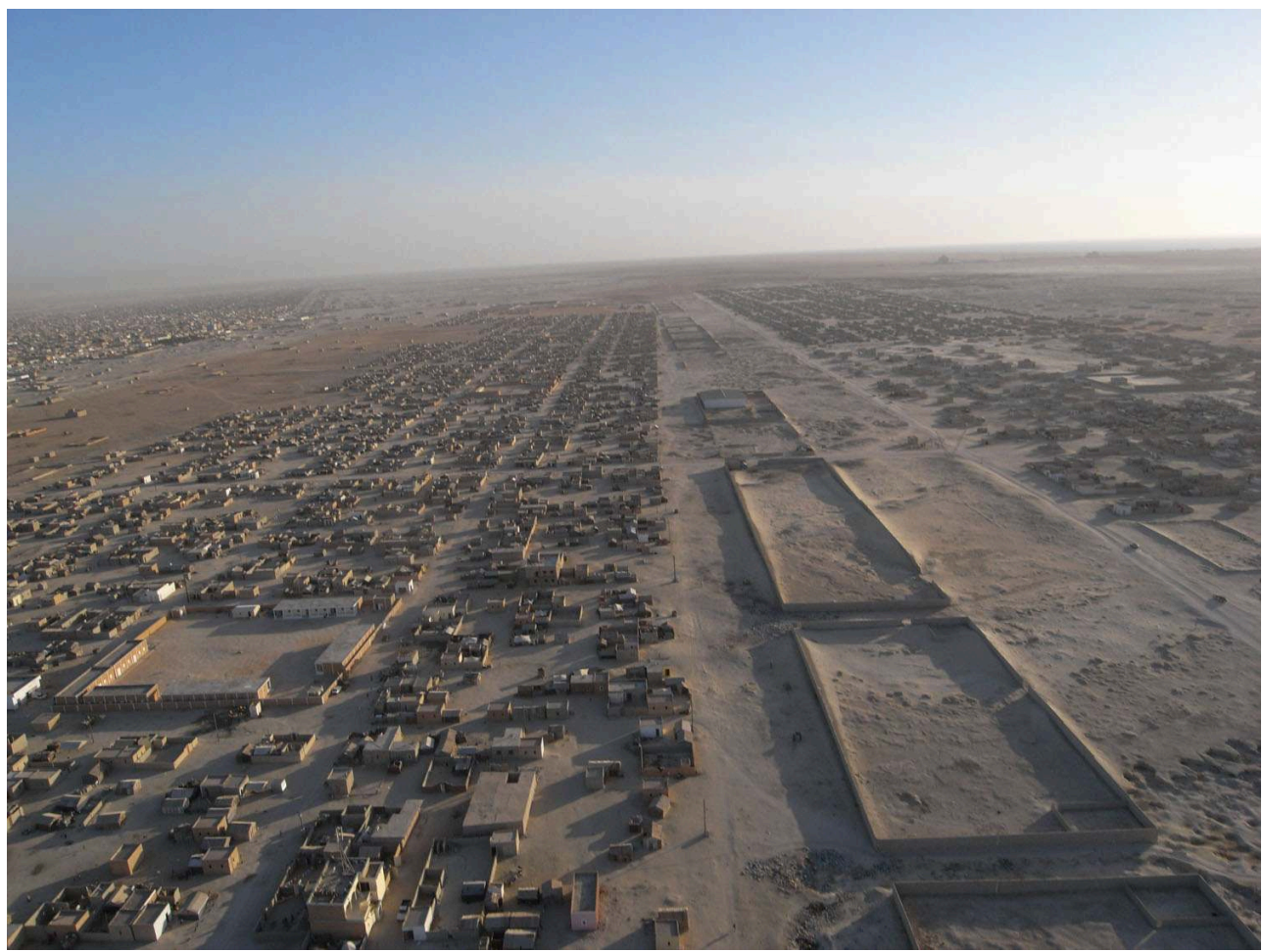

Cliché « En Haut! », Tous droits réservés, Nouakchott, Mauritanie, Novembre 2008

Photo 7 - Prise de vue verticale au-dessus du quartier de Dar El-Beïda (secteur 2)

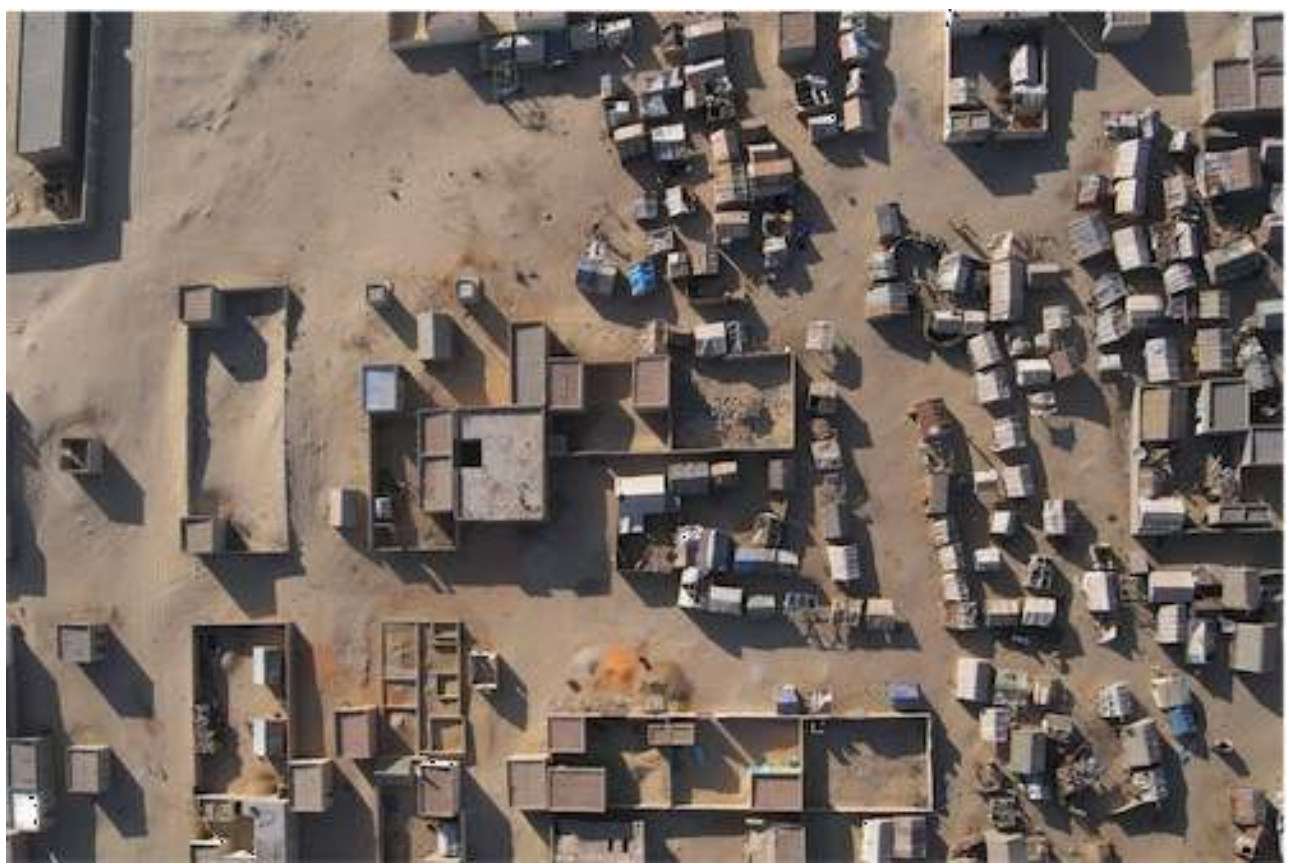

Cliché « En Haut! », Tous droits réservés, Nouakchott, Mauritanie, Novembre 2008

11 Le cerf volant est choisi en fonctions de ses caractéristiques et des conditions climatiques. Il gagne de l'altitude au fur et à mesure que la ligne est déroulée jusqu'à l'altitude souhaitée (entre 1 et 200 mètres). Il est possible de se déplacer et d'effectuer 
des prises de vues durant toute la durée du vol. 20 à 30 minutes sont suffisantes pour couvrir deux ou trois zones proches. Il est préférable de travailler au minimum à deux personnes : la première maitrisant les techniques de vol et l'autre la prise de vue et le cadrage à distance. Des talkies-walkies peuvent être utilisés pour communiquer entre la personne tenant le cerf-volant et celui qui suit le parcours, de façon à orienter le trajet. Il est à noter que la présence d'un grand cerf volant coloré dans le ciel de quartiers populaires, a systématiquement suscité curiosité et enthousiasme. Si la foule gêne parfois les manœuvres, l'échange avec les populations est riche, surtout lorsqu'un travail d'enquête a été mené en amont.

Pour ce qui est de l'équipement en tant que tel, notons qu'il n'existe pas de "kit » vendu en l'état permettant s'adonner à de la photographie aérienne sous cerf-volant. Suivant l'objectif et la fréquence de l'utilisation, il faut compter entre 500 et $1800 €$ pour un équipement complet ${ }^{3}$.

\section{Traitement et analyse des images}

Les photos prises par l'appareil nécessitent un important travail de sélection en aval ${ }^{4}$, car en moyenne, seule une photo sur quatre est exploitable, et pour celles retenues, elles ne le sont pas directement pour l'étude. Plusieurs étapes se succèdent :

- Tout d'abord, il convient d'atténuer les déformations des images liées à l'objectif. Il existe pour cela de nombreux logiciels de traitements de photo, payants ou gratuits. En ce qui nous concerne, nous avons fait le choix de "ShiftN », un logiciel gratuit permettant un grand nombre de réglages et de corrections.

- Dans un second temps, afin d'atténuer les déformations des images et donc faciliter le calage, on assemble plusieurs images. Une photo, telle la photo 7, couvre initialement une surface de $110 \mathrm{~m}$ par $80 \mathrm{~m}$. Aussi, pour couvrir de manière optimale un secteur de $150 \mathrm{~m}$ par 150 mètres, on peut utiliser jusqu'à 8 clichés assemblés ( $C f$. figures 6-8-10). L'assemblage est rendu possible grâce à plusieurs logiciels professionnels de traitement d'image et de montage panoramique, tels que Autopano pro 2.0 (100€); d'autres logiciels, gratuits, permettent d'effectuer les principales manipulations (Microsoft Composite Image Editor par exemple). Les images sont superposées suivant les zones se recoupant et assemblées en une seule photographie finale. Ces logiciels ont l'avantage d'être très faciles d'usage. Ils reconstituent le tissu urbain de manière cohérente, malgré les différences d'angles et d'altitudes de prises de vues. Certes, la rigueur ne saurait être comparée à celle exigée pour un usage dans un système d'informations géographiques (SIG) : le géo-référencement demeure approximatif, l'image n'est pas assez redressée. Néanmoins, l'image est là et exploitable, à moindre coût. Les photos enregistrent en effet tous les détails des constructions et permettent d'avoir une connaissance précise des habitations, voire des modes de vie, avant même de pénétrer dans l'enceinte de l'espace privé lors des relevés effectués au sol.

- Par la suite, un travail cartographique doit être mené. Il consiste à superposer ces nouvelles images aux photographies aériennes de 2000 : pour cela des logiciels du type d'Adobe Illustrator, ou Adobe Photoshop sont requis. Ce travail est nécessaire pour ensuite cartographier les modules et distinguer ceux construits avant 2000 et entre 2000 et 2008. Des cartes d'évolutions du bâti peuvent ainsi être élaborées (Cf. figures 11-14).

- Le dernier temps est donc celui du contrôle in situ par vérification systématique. Ce travail consiste à faire du porte à porte en rentrant dans chaque parcelle figurant sur la photographie pour interviewer les individus qui y résident : qui est propriétaire de la 
parcelle? Qui est locataire ? Où sont les modules Twize ? Fastidieux, cet ultime repérage n'en est pas moins l'occasion d'enrichir l'étude par des informations d'ordre plus qualitatif sur les quartiers. Les images deviennent ainsi le support de données plus précises sur les quartiers photographiés.

\section{Voir la ville d'EN HAUT ! pour comprendre les dynamiques d'en bas}

\section{Visualiser les mutations urbaines}

14 Nous proposons ici de nous rendre dans le quartier de Dar El-Beïda, situé en zone périphérique sud de Nouakchott pour rendre compte des changements morphologiques survenus avec et sans l'aide du programme Twize. Au travers de ces photos, on lit parfaitement les logiques venues « d'en haut », pensées par les autorités qui souhaitent légaliser et surtout contrôler ces franges urbaines, radicalement opposées à celles des habitants qui développent «en bas» des stratégies de contournement, certaines tactiques et des «compétences citadines» (Berry, Deboulet, 2000) pour s'approprier leur territoire et façonner leur quartier.

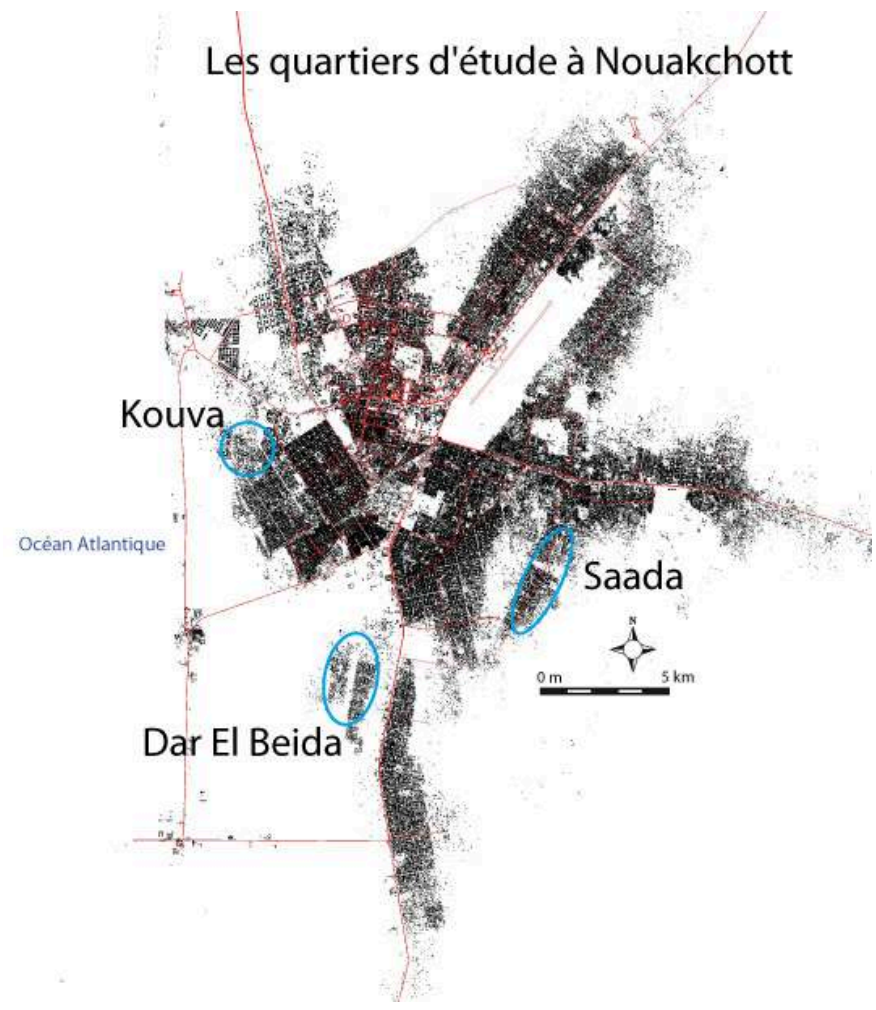

15 Source : UMR PRODIG CNRS 8586 


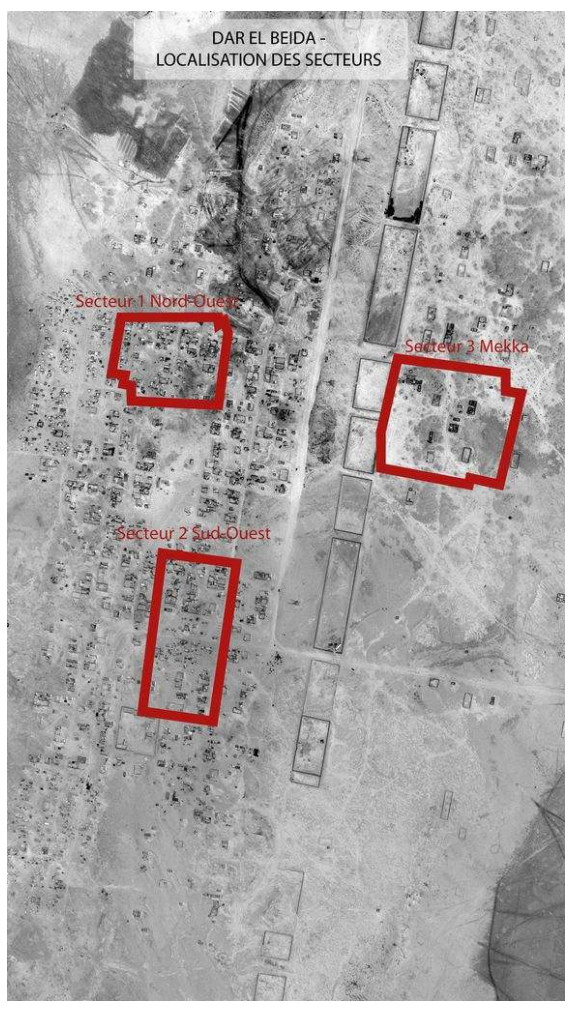

Source : UMR PRODIG CNRS 8586

16 Au sein de Dar El-Beïda, quartier caractérisé par une faible densité, l'éloignement par rapport au centre (près de $10 \mathrm{~km}$ ) et l'enclavement (jusqu'à novembre, il n'était relié au reste de l'agglomération que par une mauvaise piste), trois secteurs ont été délimités et une série de photos a été traitée et mise en perspective avec celles de 2000.

\section{Figures 5 et 6}

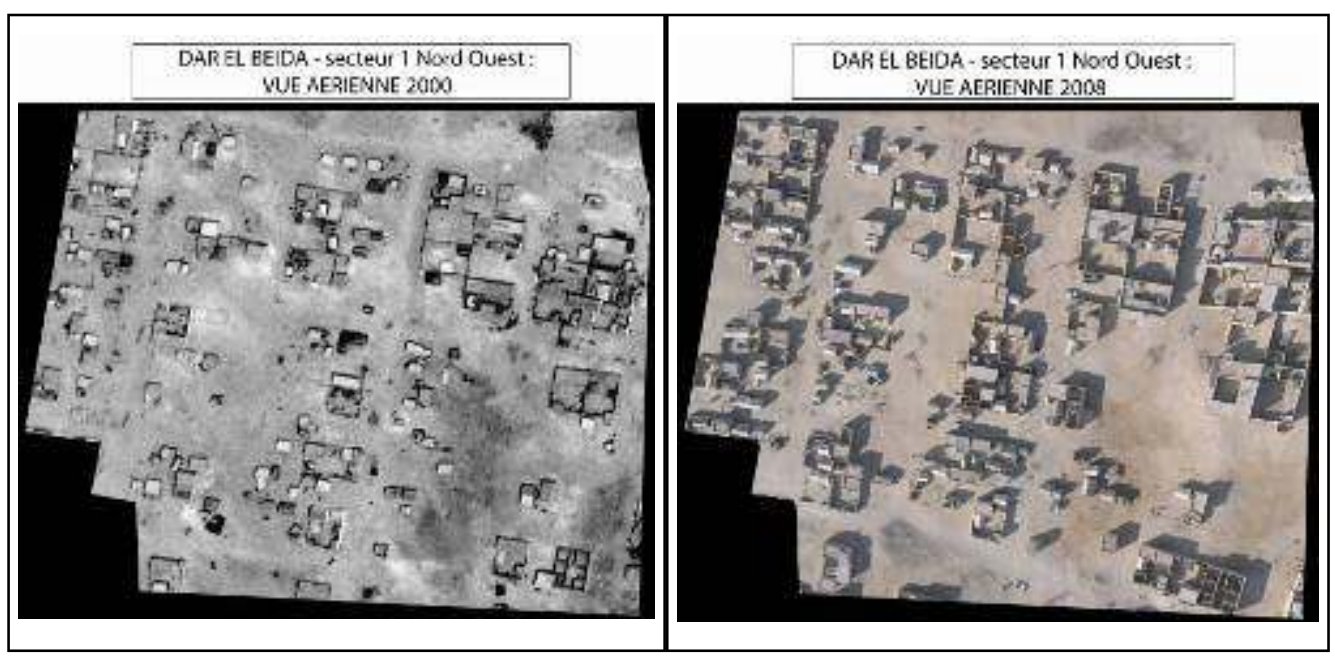

Sources : UMR PRODIG CNRS 8586 et Collectif « En Haut! », tous droits réservés

17 - Le secteur 1 correspond au vieux quartier d'intervention du GRET. Certains individus résident là depuis les années 1990. En l'espace de 8 ans, on constate que des parcelles 
(dont la taille moyenne est de $120 \mathrm{~m}^{2}$ ) se sont construites, d'autres se sont densifiées. De nombreux interstices ont été comblés.

Figures 7 et 8

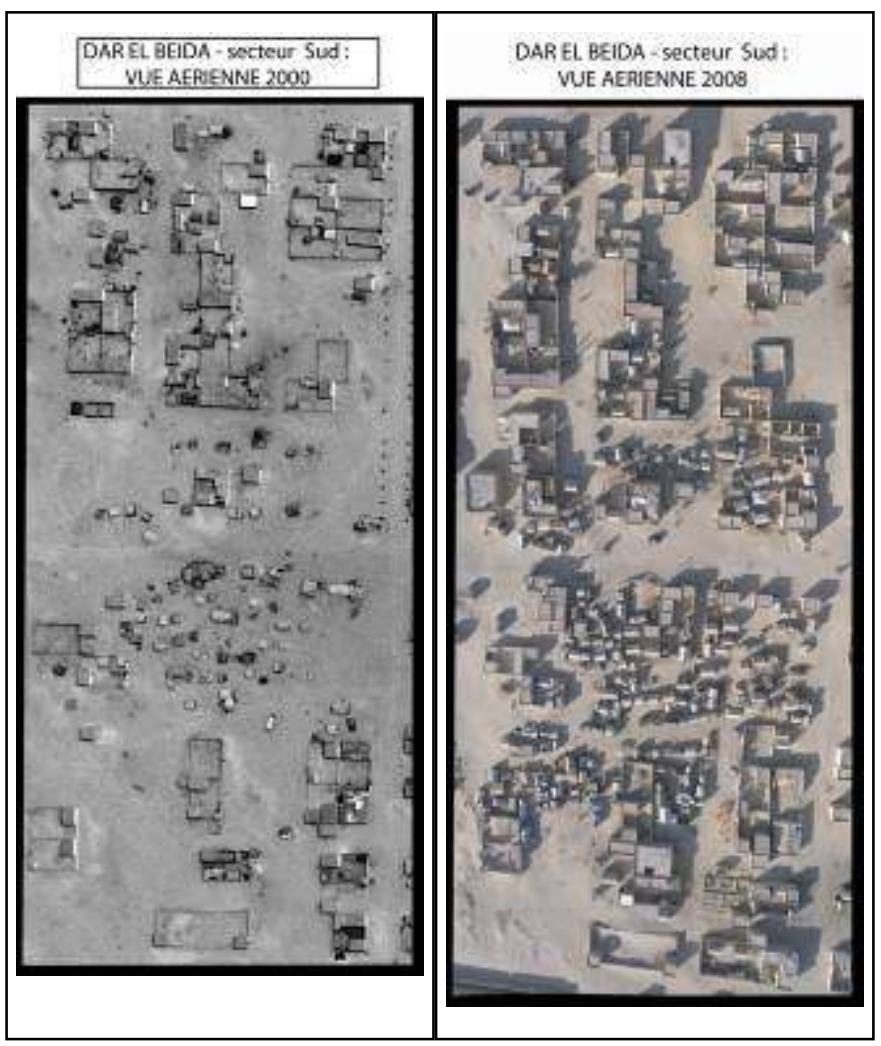

Sources : UMR PRODIG CNRS 8586 et Collectif « En Haut ! », tous droits réservés

Le secteur 2 a la particularité de ne pas être entièrement loti. On devine que dans la partie sud, le gouvernement n'a pas encore distribué les terrains. Cela se voit d'autant plus que le nombre de baraques a considérablement augmenté, alors que dans la partie plus au nord, l'habitat s'est densifié. Très clairement, on assiste à un phénomène de squatt, localement appelé gazra. Les habitants occupent illégalement l'espace en installant une baraque, en bois, dans l'attente que les pouvoirs publics se décident à régulariser cet espace (Cf. Photo 6). Si enregistrement il $\mathrm{y}$ a, ils espèrent se voir attribuer une parcelle. 


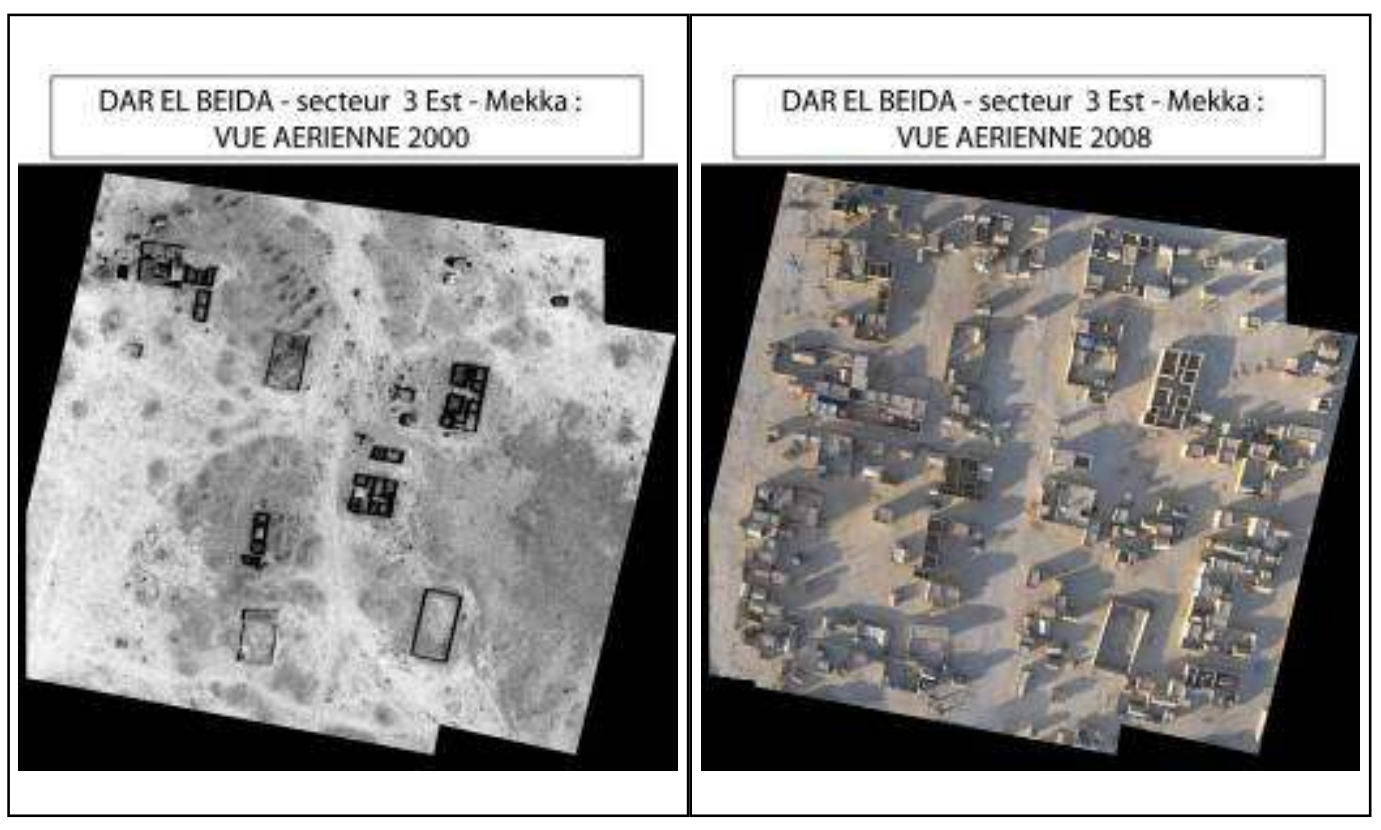

Sources : UMR PRODIG CNRS 8586 et Collectif «En Haut ! », tous droits réservés

- Le secteur 3 n'a été aménagé que récemment. L'espace était quasi inoccupé il y a peu de temps encore. Des habitants qui vivaient initialement dans un bidonville de Nouakchott y ont été recasés en 2006, dans le cadre d'un vaste projet de restructuration urbaine. La construction et la densification se sont donc faites dans un laps de temps relativement court (2006-2008).

\section{Mesurer l'impact d'un programme de développement}

Les termes de références de l'étude demandaient de calculer l'impact du programme dans ces quartiers ; par conséquent, nous avons cherché à mettre au point une méthode simple qui permettait de faire ressortir quelques chiffres, certes peu nombreux, mais néanmoins parlants. C'est là un apport considérable au regard des données statistiques ou mêmes chiffrées qui font complètement défaut dans le pays. Dans la mesure où le programme Twize du GRET offrait la possibilité de construire une chambre de $20 \mathrm{~m}^{2}$ ou une clôture+une latrine (dont le prix est équivalent à un module chambre), nous avons choisi le mode de dénombrement suivant:

$$
\begin{aligned}
& \text { - } \text { une chambre }=1 \\
& \text { - } \text { - une clôture }=0,5 \\
& \text { - } \text { - une latrine }=0,5
\end{aligned}
$$

Ce choix permet de compter sur chaque parcelle le nombre de modules, et par extension dans chaque zone le nombre de module. Après décompte, un relevé systématique a été effectué sur le terrain comme expliqué ci-dessus. A partir de ces données et des changements observés entre 2000 et 2008, il fut possible de réaliser les figures suivantes. Les modules construits grâce à l'aide du programme figurent en rose pour ceux qui existaient en 2000 et en rouge pour ceux qui ont été construits entre 2000 et 2008 . 
Figure 11
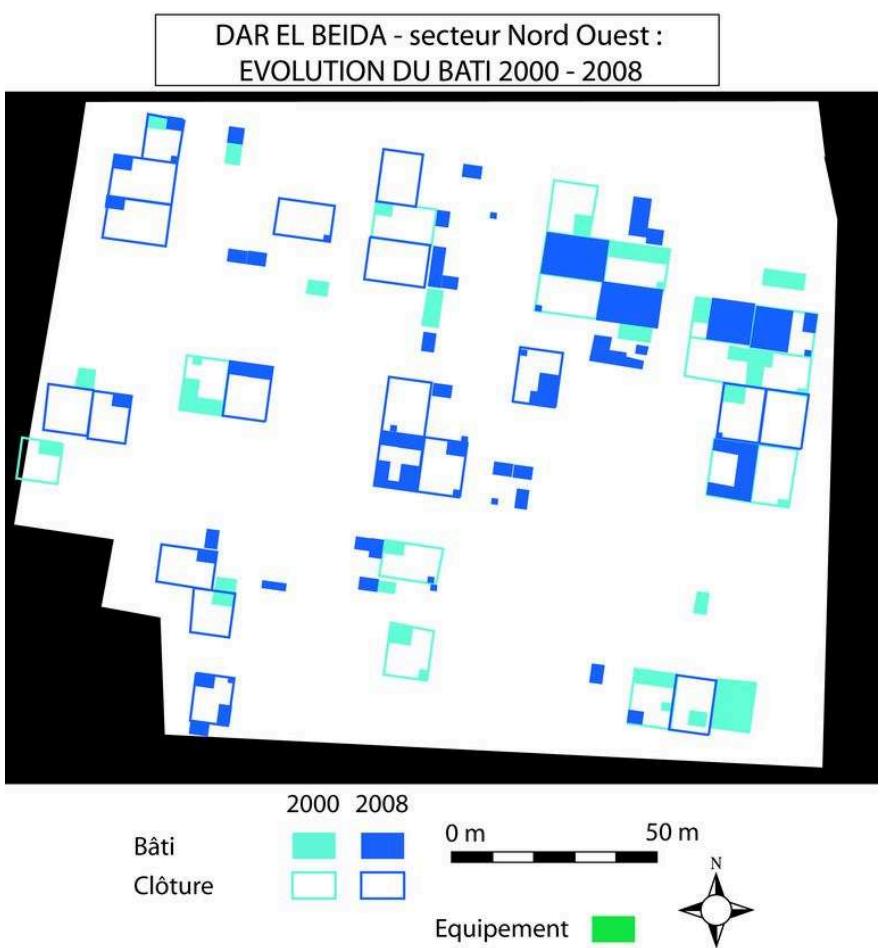

Figure 12

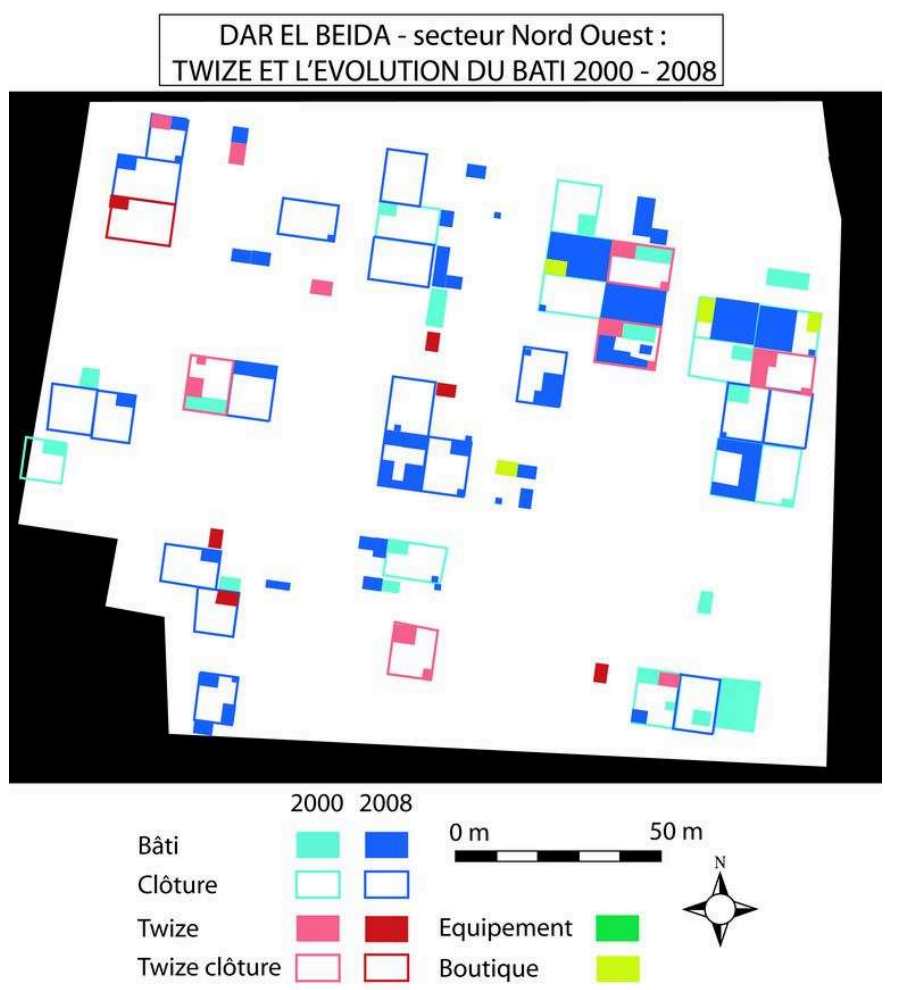

Cartographie et traitement image : B. Bosselut, novembre 2008. 
Figures 13 et 14

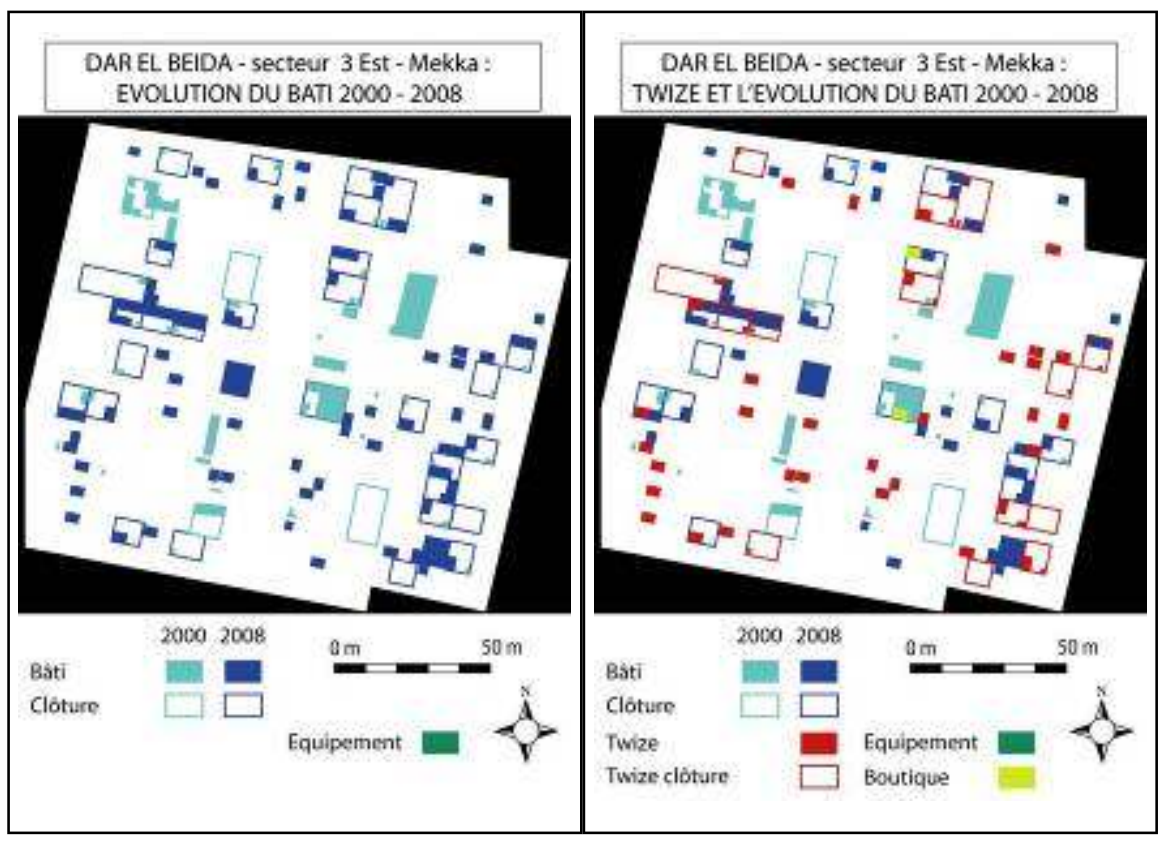

Cartographie et traitement image : B. Bosselut, novembre 2008.

Ce jeu de cartes permet de lire deux impacts différenciés du programme Twize dans deux quartiers pourtant proches spatialement :

- l'impact du programme est moindre et relativement diffus dans le secteur 1 . Après décompte et relevés on constate que :

- les constructions ont été multipliées par 3 entre 2000 et 2008

24 - 40\% des parcelles sont bâties en 2000 contre $70 \%$ en 2008

25 - mais seulement $17 \%$ des constructions relèvent du programme Twize

- 15 des 59 parcelles bâties ont bénéficié d'un module Twize, soit 25\%

- l'impact de Twize est bien plus important dans le secteur 3 puisque :

27 - les constructions ont été multipliées par 5

28 - le nombre de parcelles construites a été multiplié par 8 et le nombre de modules par 6 .

$29-30 \%$ des constructions relèvent du programme Twize

$30-42$ des 74 parcelles construites dans le quartier ont bénéficié du programme, soit $58 \%$

\section{Les prises de vue verticales comme outil de mesure des transformations des} parcelles 


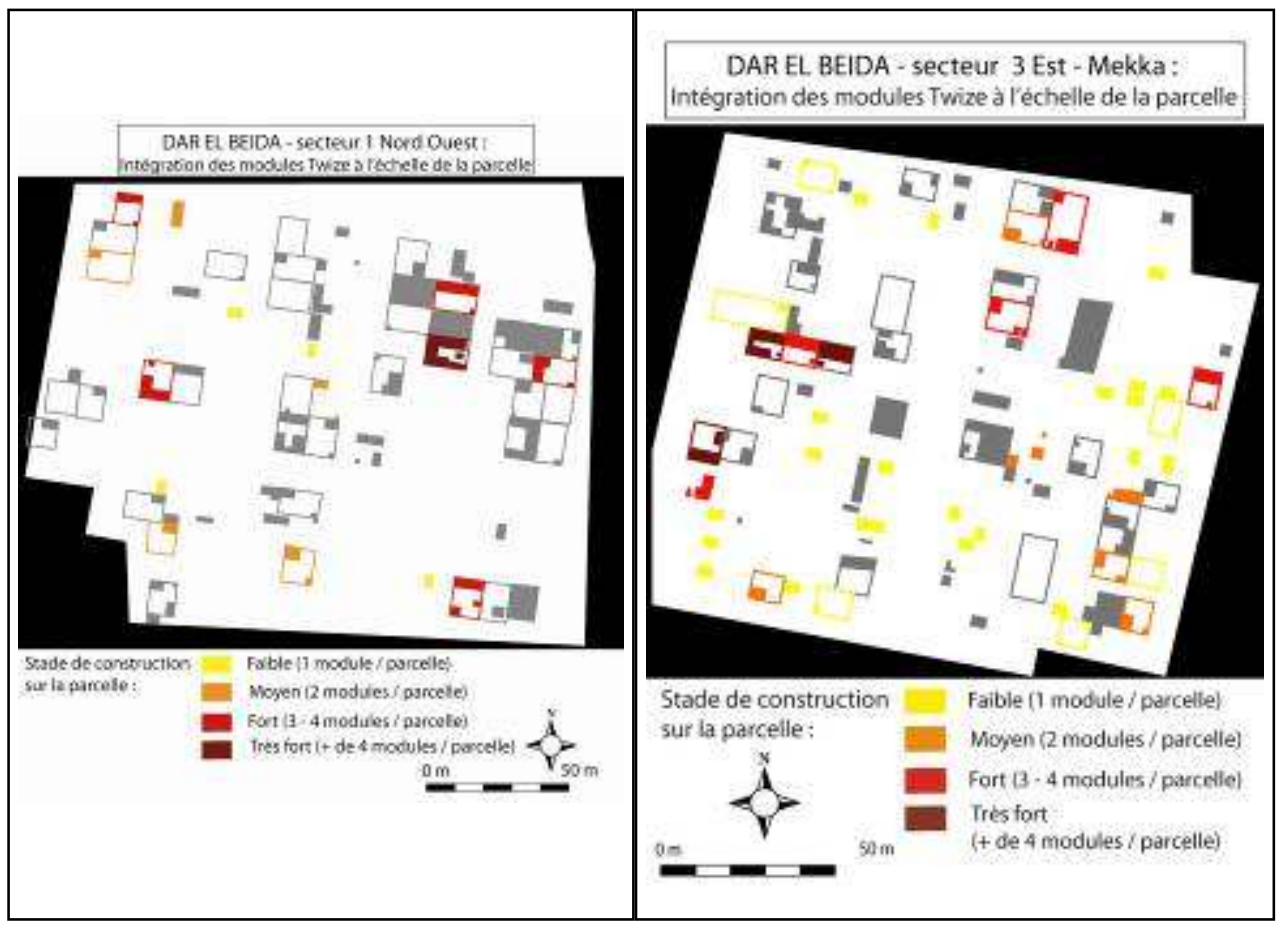

Montages et cartographie : Benjamin Bosselut, décembre 2008

Figure 17

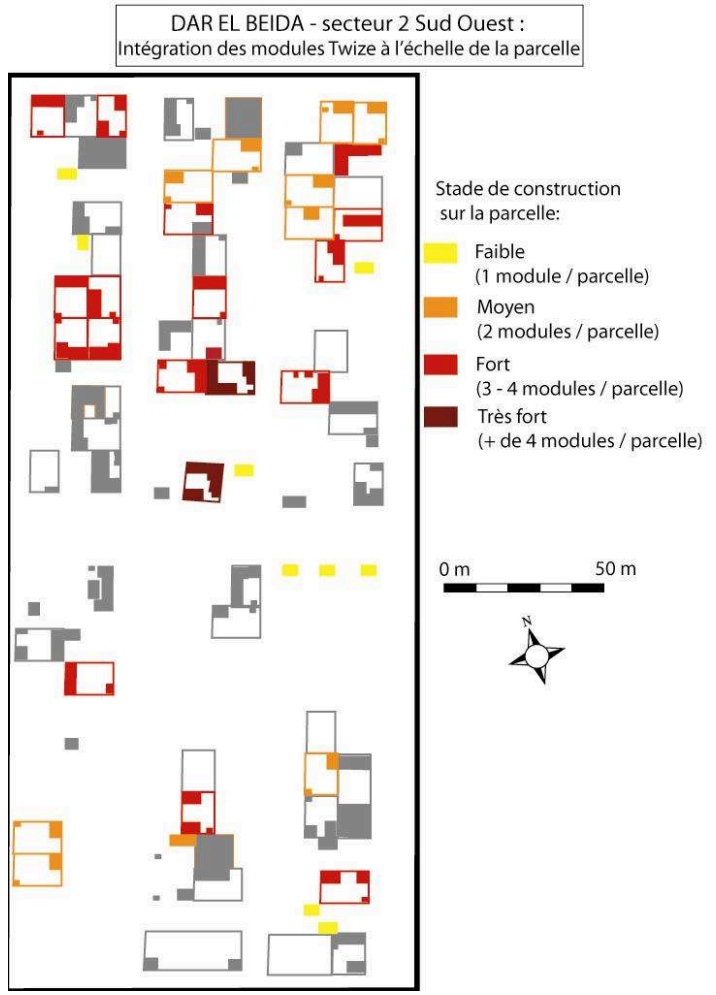

31 Outre les données concernant le nombre de parcelles construites directement par le programme, la prise de vue verticale permet de voir ce que les ménages ont construit 
au sein de leur parcelle depuis une dizaine d'années. Ce point constitue un apport essentiel en Mauritanie : à cause des hauts murs traditionnels, il est généralement malaisé de discerner le nombre de constructions sur une parcelle sans pouvoir y pénétrer. Les photos aériennes permettent de dépasser ce problème puisqu'elles offrent la possibilité de visualiser les changements au sein de chaque entité. Un constat simple peut alors être établi : plus les ménages sont installés depuis longtemps, plus ils ont eu le temps et l'opportunité de construire, donc d'intégrer le module à la parcelle. Les images permettent encore de démontrer que le programme Twize est considéré comme un point de départ permettant une amélioration générale des conditions de vie. A la suite de la construction d'un premier module, les individus ont en effet tendance à poursuivre la construction (en moyenne deux modules sur les anciennes parcelles comme dans le secteur 1 et un pour l'instant dans la zone de recasement du secteur 3)

\section{Les prises de vue obliques ou comment suivre le front d'urbanisation}

Photo 8 - Vue du sud-ouest de Nouakchott à proximité du littoral

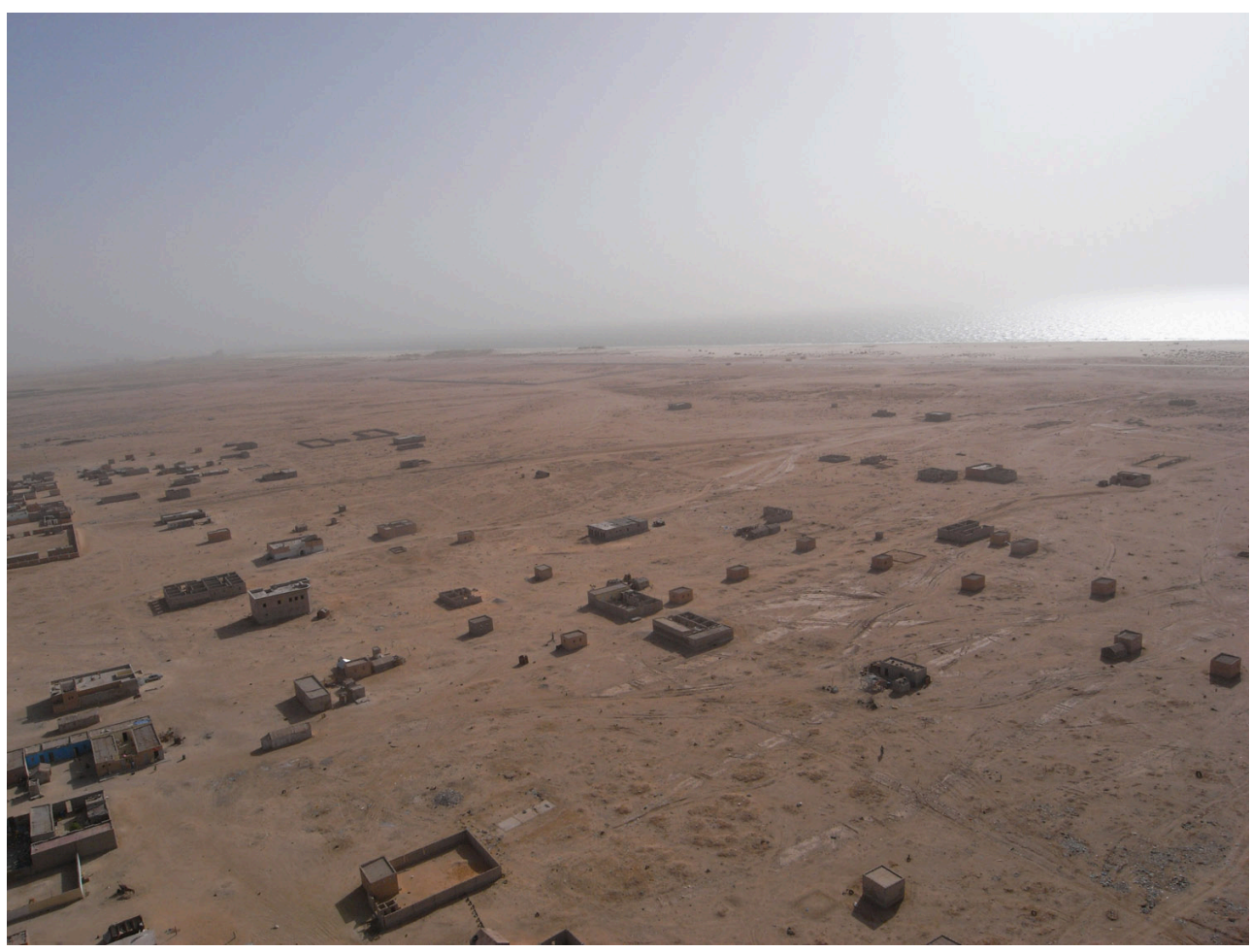

Cliché «En Haut ! », Tous droits réservés, Nouakchott, Mauritanie, Novembre 2008

Les photos assistées par cerf-volant offrent la possibilité d'observer les fronts d'urbanisation (photos 1,2,3), ce qui a permis de mettre en lumière des processus insoupçonnés. A la vue de cette image, on peut deviner la présence de modules Twize isolés. Nulle clôture, nulle cuisine ni latrine, nul signe de densification ou d'intégration qui laisserait supposer une forme de territorialité et d'ancrage. Les Twize elles-mêmes semblent inhabitées ou abandonnées. Ici, le programme semble avoir participé à la progression de franges urbaines et même à une certaine forme de spéculation. Pareille photo laisse à penser que certaines personnes plus aisées auraient profité du programme pour habiller leur parcelle, les valoriser en attendant que des infrastructures (eau, électricité) soient installées par les pouvoirs publics. Après 
enquêtes sur place, ces chambres se sont révélées inoccupées ou louées, et effectivement la propriété d'individus plus aisés, non visés initialement par le programme. Nous avons alors pu tirer et localiser les informations suivantes :

Photo 9

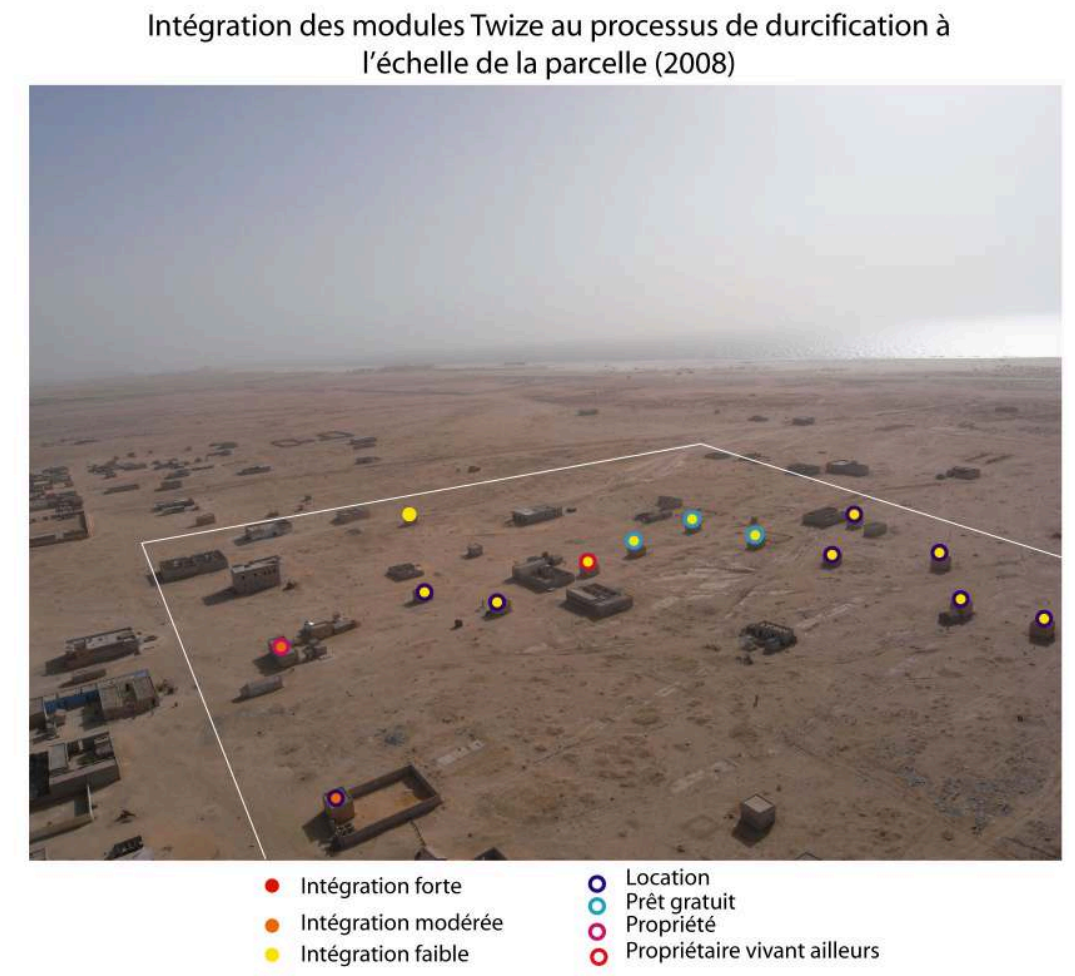

Cliché « En Haut ! », Tous droits réservés, Nouakchott, Mauritanie, Novembre 2008 Traitement image B. Bosselut.

La photographie aérienne par cerf-volant qui existe depuis plus d'un siècle est fréquemment utilisée en archéologie mais son fort pouvoir démonstratif pour ce qui est des processus urbains ne demande qu'à être exploré. Au-delà des quelques chiffres qui ont pu être tirés dans le cadre de l'étude d'impact et du cas mauritanien, c'est un outil facilement utilisable qui permet d'être à la fois en haut et en bas et propose une lecture sensible du paysage. En ce qui concerne les villes du Sud en particulier, dont on ne sait généralement pas grand chose ou du moins jamais en tant réel tant leurs évolutions sont rapides, c'est là un outil de visualisation mais également d'analyse particulièrement efficace.

\section{BIBLIOGRAPHIE}

ALES J.M. et TAGALA D., 1985, « La photo aérienne sans avion », Archéologia, 179. p. 53-58. 
ANDERSON R.C. 1979, « A kite supported system for remote aerial photography », Aerial Archaeology. 4. p. 4-11.

BATUT A., 1890, La photographie aérienne par cerf volant, Paris : Gauthier Villars. 74 p.

BERRY-CHIKHAOUI I., DEBOULET A. (dir.), 2000, Les compétences des citadins dans le monde arabe : penser, faire et transformer la ville, Paris : Karthala ; Tours : Urbama ; Tunis : IRMC. 406 p.

CHOPLIN A., 2006, « Le foncier urbain en Afrique : entre informel et rationnel, l'exemple de Nouakchott, Mauritanie », Les Annales de géographie, n 647, p. 69-91.

CHOPLIN A., 2009 (sous presse), Nouakchott, au carrefour de la Mauritanie et du monde, Paris : Karthala, 367 p.

CHOPLIN A., BOSSELUT B. PEPIN A., 2009, Etude d'impact du programme Twize, Université Paris-Est Marne-la-vallée, $174 \mathrm{p}$.

CREUSOT A.-C., 2002, Financement de l'habitat social en Mauritanie : l'expérience du programme Twize, Ville en développement, $\mathrm{n}^{\circ} 56,4 \mathrm{p}$.

GRET, 2003, Programme Twize, Manuel de procédures, Nouakchott : RIM-CDHLCPI, 266 p.

TIELKES E., 2003, L'œil du cerf-volant. Évaluation et suivi des états de surface par photographie aérienne sous cerf-volant, ICRISAT et université de Hohenheim, Allemagne, $113 \mathrm{p}$.

THENOT A., 2007, Modèles de données pour l'appréhension et la gestion des risques à Nouakchott (Mauritanie), Une capitale contre vents et marées, Thèse doctoraant en géographie à l'Université de Paris $1,538 \mathrm{p}$.

Entre de multiples sites, voir également :

http://archeokap.free.fr/

http://photocerfvolant.free.fr

http://www.kapshop.com/

http://arch.ced.berkeley.edu/kap/

http://pagesperso-orange.fr/kap-chagny/index.html

http://kap.nonsenz.org

\section{NOTES}

1. Cf. www.gret.org

2. Discussion en septembre 2008 au siège du GRET avec Philippe Lavigne-Delville, alors directeur scientifique du GRET. Voir les termes de références en annexe de l'étude d'impact (Choplin, Bosselut, Pépin, 2009)

3. L'équipement comprend un cerf-volant qui peut coûter entre $150 €$ (fabrication maison) et $250 €$ (acheté prêt à voler). Il vaut mieux disposer de plusieurs modèles (au moins 3 pour pouvoir s'adapter aux conditions climatiques). Les prix de l'appareil photographique numérique varient entre $200 €$ et $1000 €$ (et plus pour du matériel professionnel). Pour la nacelle et la télécommande, compter entre $100 €$ pour une fabrication maison et $800 €$ pour une nacelle prête à l'usage et avec retour vidéo). Le retour vidéo vaut à lui seul $300 €$.

4. Notons qu'au moment de l'étude, l'équipe n'était pas munie d'un retour vidéo. Celui-ci peut renvoyer l'image sur un petit écran fixé sur la télécommande, ce qui permet d'optimiser le cadrage et de réduire le travail de tri des images. 


\section{RÉSUMÉS}

Cet article démontre l'utilité de la photographie aérienne sous cerf-volant pour les études urbaines, et en particulier pour les villes du Sud qui connaissent une croissance rapide. Cette technique est testée à Nouakchott (Mauritanie) dans le cadre d'une étude d'impact d'un programme d'habitat social. L'absence de données cartographiques actualisées est ainsi compensée par cet outil qui permet de voir l'évolution de la ville en temps réel. Ce texte explique les différentes manières d'utiliser ces photos et étapes pour produire des données exploitables.

This paper shows how the kite aerial photography can be used in urban studies, relying on a concrete application experience for a social housing program in Nouakchott (Mauritania). Particularly, it is useful in order to study Southern cities involved by urban sprawl. This tool allows to see urban developments "in real-time " and should compensate the lack of mapping up-to-date data. This article explains how we can use kite pictures and technical procedures to produce exploitable data.

\section{INDEX}

Mots-clés : ville, sud, photographie aérienne sous cerf-volant, études urbaines, habitat social, Mauritanie, Nouakchott

Keywords : city, south, kite aerial photography, urban studies, social housing, Mauritania, Nouakchott

\section{AUTEURS}

\section{BENJAMIN BOSSELUT}

Benjamin Bosselut est diplômé en $3^{\mathrm{e}}$ année de magister d'urbanisme et aménagement du territoire à l'université Paris-Sorbonne.

\section{MARION BROQUÈRE}

Marion Broquère est assistante technique FIBA au Parc National du Banc d'Arguin en Mauritanie.

\section{ARMELLE CHOPLIN}

Armelle Choplin est maître de conférences en géographie, à l'Université Paris-Est Marne-lavallée. Son ouvrage « Nouakchott au carrefour de la Mauritanie et du monde » est sous presse aux éditions Karthala. Cet ouvrage est édité par l'UMR PRODIG, laboratoire au sein duquel Armelle Choplin a réalisé son doctorat et auquel elle est membre associé. En 2008, elle a déjà rédigé un article pour EchoGéo intitulé « La Mauritanie à l'épreuve de l'islamisme et des menaces terroristes ", URL : http://echogeo.revues.org/document4363.html. Dans le numéro 114 de Politique africaine spécial Mauritanie sorti en juin 2009, elle a publié, avec Jérôme Lombard, « La Mauritanie offshore. Extraversion économique, Etat et sphères dirigeantes », p. 85-102.

\section{SIMON NANCY}

Simon Nancy est consultant UICN/ Assistant technique PoWPA a la Direction des Aires Protégées et du Littoral, MEDD, en Mauritanie. Il anime avec Marion Broquère le collectif « en Haut ! : 
photographie aérienne par cerf-volant et communication territoriale. Pour visualiser quelques unes des photographies, voir http://www.mauritanie-decouverte.net/nouakchott_vue_du_ciel/ index.html 